\title{
BEST CONSTANTS FOR TWO NONCONVOLUTION INEQUALITIES
}

\author{
MICHAEL CHRIST AND LOUKAS GRAFAKOS
}

(Communicated by J. Marshall Ash)

\begin{abstract}
The norm of the operator which averages $|f|$ in $L^{p}\left(\mathbb{R}^{n}\right)$ over balls of radius $\delta|x|$ centered at either 0 or $x$ is obtained as a function of $n, p$ and $\delta$. Both inequalities proved are n-dimensional analogues of a classical inequality of Hardy in $\mathbb{R}^{1}$. Finally, a lower bound for the operator norm of the Hardy-Littlewood maximal function on $L^{p}\left(\mathbb{R}^{n}\right)$ is given.
\end{abstract}

\section{INTRODUCTION}

A classical result of Hardy [HLP] states that if $f$ is in $L^{p}\left(\mathbb{R}^{1}\right)$ for $p>1$, then

$$
\left(\int_{0}^{\infty}\left(\frac{1}{x} \int_{0}^{x}|f(t)| d t\right)^{p} d x\right)^{1 / p} \leq \frac{p}{p-1}\left(\int_{0}^{\infty}|f(t)|^{p} d t\right)^{1 / p}
$$

and the constant $p /(p-1)$ is the best possible. By considering two-sided averages of $f$ instead of one-sided, $(0.1)$ can be equivalently formulated as:

$$
\left(\int_{-\infty}^{\infty}\left(\frac{1}{2|x|} \int_{-|x|}^{|x|}|f(t)| d t\right)^{p} d x\right)^{1 / p} \leq \frac{p}{p-1}\left(\int_{-\infty}^{\infty}|f(t)|^{p} d t\right)^{1 / p} .
$$

We denote by $D(a, R)$ the ball of radius $R$ in $\mathbb{R}^{n}$ centered at $a$. Let $(T f)(x)$ be the average of $|f| \in L^{p}\left(\mathbb{R}^{n}\right)$ over the ball $D(0,|x|)$. The analogue of $(0.2)$ for $\mathbb{R}^{n}$ is the inequality:

$$
\|T f\|_{L^{p}} \leq C_{p}(n)\|f\|_{L^{p}}
$$

for some constant $C_{p}(n)$ which depends a priori on $p$ and $n$. Our first result is that the best constant $C_{p}(n)$ which satisfies $(0.3)$ for all $f \in L^{p}\left(\mathbb{R}^{n}\right)$ is $p^{\prime}=p /(p-1)$, the same constant as in dimension one. Another version of Hardy's inequality in $\mathbb{R}^{n}$ with the best possible constant can be found in [F].

Next we consider a similar problem. An equivalent formulation of $(0.1)$ and $(0.2)$ is

$$
\left(\int_{-\infty}^{\infty}\left(\frac{1}{2|x|} \int_{x-|x|}^{x+|x|}|f(t)| d t\right)^{p} d x\right)^{1 / p} \leq \frac{p}{2^{1 / p}(p-1)}\left(\int_{-\infty}^{\infty}|f(t)|^{p} d t\right)^{1 / p},
$$

Received by the editors May 5, 1993 and, in revised form, September 3, 1993.

1991 Mathematics Subject Classification. Primary 42B25.

The authors' research was partially supported by the National Science Foundation. 
where $f$ is in $L^{p}\left(\mathbb{R}^{1}\right)$. Let $(S f)(x)$ be the average of $|f| \in L^{p}\left(\mathbb{R}^{n}\right)$ over the ball $D(x,|x|)$. We compute the operator norm $c_{p, n}$ of $S$ on $L^{p}\left(\mathbb{R}^{n}\right)$ as a function of $n$ and $p$. The precise value of the constant $c_{p, n}$ is given in Theorem 2.

In section 3 a lower bound for the operator norm of the Hardy-Littlewood maximal function on $L^{p}\left(\mathbb{R}^{n}\right)$ is given. Finally, in section 4 the norm on $L^{p}\left(\mathbb{R}^{n}\right)$ of the operator which averages $f$ over the ball of radius $\delta|x|$ centered at either 0 or $|x|$ is given as a function of $\delta, p$, and $n$, for any $\delta>0$.

Throughout this note, $\omega_{n-1}$ will denote the area of the unit sphere $S^{n-1}$ and $v_{n}$ the volume of the unit ball in $\mathbb{R}^{n}$.

\section{HARDY'S INEQUALITY ON $\mathbb{R}^{n}$}

In this section we will prove inequality $(0.3)$ with constant $C_{p}(n)=p^{\prime}=$ $p /(p-1)$. We denote by $|A|$ the Lebesgue measure of the set $A$ and by $\chi_{A}$ its characteristic function.

Theorem 1. Let $f \in L^{p}\left(\mathbb{R}^{n}\right)$, where $1<p<\infty$. The following inequality holds:

$$
\left(\int_{\mathbb{R}^{n}}\left(\frac{1}{|D(0,|x|)|} \int_{D(0,|x|)}|f(y)| d y\right)^{p} d x\right)^{1 / p} \leq \frac{p}{p-1}\left(\int_{\mathbb{R}^{n}}|f(y)|^{p} d y\right)^{1 / p},
$$

and the constant $p^{\prime}=p /(p-1)$ is the best possible.

Proof. Fix $f \in L^{p}\left(\mathbb{R}^{n}\right)$. Without loss of generality, assume that $f$ is nonnegative and continuous. Let $\mathbb{R}^{+}$denote the multiplicative group of positive real numbers with Haar measure $\frac{d t}{t}$. The function $t^{n / p^{\prime}} \chi_{[0,1]}$ is in $L^{1}\left(\mathbb{R}^{+}, \frac{d t}{t}\right)$ with norm $p^{\prime} / n$. For a fixed $\theta$ in the unit sphere $S^{n-1}$, the function $t \rightarrow f(t \theta) t^{n / p}$ is in $L^{p}\left(\mathbb{R}^{+}, \frac{d t}{t}\right)$. The group inequality $\|g * K\|_{L^{p}} \leq\|g\|_{L^{p}}\|K\|_{L^{1}}$ gives:

$$
\int_{r=0}^{\infty}\left(\int_{0}^{1} f(r t \theta)(r t)^{\frac{n}{p}} t^{\frac{n}{p^{\prime}}} \frac{d t}{t}\right)^{p} \frac{d r}{r} \leq\left(\int_{r=0}^{\infty}\left(f(r \theta) r^{\frac{n}{p}}\right)^{p} \frac{d r}{r}\right)\left(\frac{p^{\prime}}{n}\right)^{p} .
$$

Note that equality holds in (1.2) if and only if equality holds in $\|g * K\|_{L^{p}} \leq$ $\|g\|_{L^{p}}\|K\|_{L^{1}}$. This happens in the limit by the sequence $g_{\epsilon, N}=\chi_{[\epsilon, N]}$. Since $g(t)=f(t \theta) t^{n / p}$, we conclude that equality is attained in (1.2) in the limit by the sequence

$$
f_{\epsilon, N}(t \theta)=t^{-n / p} \chi_{\epsilon \leq t \leq N} \quad \text { as } \epsilon \rightarrow 0 \text { and } N \rightarrow \infty .
$$

Note that $T f$ is a radial function. Expressing all integrals in polar coordinates, we reduce (1.1) to a convolution inequality on the multiplicative group $\mathbb{R}^{+}$. We have

$$
\begin{aligned}
\|T f\|_{L^{p}\left(\mathbb{R}^{n}\right)}^{p} & =\omega_{n-1} \int_{r=0}^{\infty}\left(\frac{1}{v_{n} r^{n}} \int_{t=0}^{r} \int_{\theta \in S^{n-1}} f(t \theta) t^{n-1} d \theta d t\right)^{p} r^{n-1} d r \\
& =\frac{\omega_{n-1}}{v_{n}^{p}} \int_{r=0}^{\infty}\left(\int_{S^{n-1}} \int_{t=0}^{1} f(r t \theta)(r t)^{\frac{n}{p}} t^{\frac{n}{p^{p}}} \frac{d t}{t} d \theta\right)^{p} \frac{d r}{r} .
\end{aligned}
$$

We apply Hölder's inequality with exponents $\frac{1}{p}+\frac{1}{p^{\prime}}=1$ to the functions 1 and $\theta \rightarrow \int_{t=0}^{1} f(r t \theta)(r t)^{n / p} t^{n / p^{\prime}} \frac{d t}{t}$ and then to Fubini's theorem to interchange the integrals in $\theta$ and $r$. We obtain that (1.4) is bounded above by

$$
\frac{\omega_{n-1}}{v_{n}^{p}} \omega_{n-1}^{\frac{p}{p^{p}}} \int_{S^{n-1}} \int_{r=0}^{\infty}\left(\int_{t=0}^{1} f(r t \theta)(r t)^{\frac{n}{p}} t^{\frac{n}{p^{p}}} \frac{d t}{t}\right)^{p} \frac{d r}{r} d \theta .
$$


Note that if $f$ is a radial function, then (1.4) and (1.5) are identical. We now apply (1.2) to majorize (1.5) by

$$
\frac{\omega_{n-1}^{p}}{v_{n}^{p}}\left(\frac{p^{\prime}}{n}\right)^{p} \int_{S^{n-1}} \int_{r=0}^{\infty} f(r \theta)^{p} r^{n} \frac{d r}{r} d \theta=\left(\frac{p}{p-1}\right)^{p}\|f\|_{L^{p}\left(\mathbf{R}^{n}\right)}^{p}
$$

using the fact that $\omega_{n-1}=n v_{n}$. We have now obtained the inequality $\|T f\|_{L^{\nu}} \leq$ $p^{\prime}\|f\|_{L^{p}}$. Equality holds when the family of functions (1.3) is radial. Therefore, the extremal family for inequality (1.1) is $|x|^{-n / p} \chi_{\epsilon \leq|x| \leq N}$, as $\epsilon \rightarrow 0$ and $N \rightarrow \infty$.

\section{A VARIANT OF HARDY'S INEQUALITY ON $\mathbb{R}^{n}$}

The derivation of the $n$-dimensional analogue of $(0.4)$ is more subtle. Let $B(s, t)$ denote the usual beta-function $\int_{0}^{1} x^{t}(1-x)^{s} d x$. Our second result is Theorem 2. Let $1<p<\infty$ and $c_{p, n}=p^{\prime} \frac{\omega_{n-2}}{\omega_{n-1}} 2 \frac{n}{p^{\prime}}-1 B\left(\frac{1}{2}\left(\frac{n}{p^{\prime}}-1\right), \frac{n-3}{2}\right)$. The following inequality holds for all $f$ in $L^{p}\left(\mathbb{R}^{n}\right)$ :

$$
\left(\int_{\mathbb{R}^{n}}\left(\frac{1}{|D(x,|x|)|} \int_{D(x,|x|)}|f(y)| d y\right)^{p} d x\right)^{1 / p} \leq c_{p, n}\left(\int_{\mathbb{R}^{n}}|f(y)|^{p} d y\right)^{1 / p}
$$

and the constant $c_{p, n}$ is the best possible.

Proof. We use duality. Fix $f$ and $g$ positive and continuous with $\|f\|_{L^{p}\left(\mathbb{R}^{n}\right)} \leq$ 1 and $\|g\|_{L^{\prime}\left(\mathbf{R}^{n}\right)} \leq 1$. We will show that $\int g(x)(S f)(x) d x \leq c_{p, n}$. We express both $g$ and $S f$ in polar coordinates by writing $x=r \phi$ and $y=t \theta$. The relation $|x-y| \leq|x|$ is equivalent to $\theta \cdot \phi \geq t / 2 r$. We obtain

$$
\begin{array}{rl}
\int_{\mathbb{R}^{n}} & g(x)(S f)(x) d x \\
= & \int_{\mathbb{R}^{n}} \int_{\mathbb{R}^{n}} \frac{1}{v_{n}|x|^{n}} f(y) g(x) \chi_{D(x,|x|)}(y) d x d y \\
= & \frac{1}{v_{n}} \iint_{\left(S^{n-1}\right)^{2}} \int_{r=0}^{\infty} \int_{t=0}^{2 r} f(t \theta) g(r \phi) \chi_{\phi \cdot \theta \geq t / 2 r} t^{n} \frac{d t}{t} \frac{d r}{r} d \phi d \theta \\
= & \frac{2 \frac{n}{p^{n}}}{v_{n}} \iint_{\left(S^{n-1}\right)^{2}} \int_{r=0}^{\infty} g(r \phi) r r^{\frac{n}{p^{\prime}}}\left(\int_{t=0}^{1} f(2 r t \theta)(2 r t)^{\frac{n}{p}} \chi_{\phi^{*} \theta \geq t} t^{\frac{n}{p^{\prime}}} \frac{d t}{t}\right) \frac{d r}{r} d \phi d \theta \\
\leq & \frac{2 \frac{n}{p^{n}}}{v_{n}} \iint_{\left(S^{n-1}\right)^{2}} G(\phi)\left[\int_{r=0}^{\infty}\left(\int_{t=0}^{1} f(2 r t \theta)(2 r t)^{\frac{n}{p}} \chi_{\phi^{*} \theta \geq t} t^{\frac{n}{p^{n}}} \frac{d t}{t}\right)^{p} \frac{d r}{r}\right]^{1 / p} d \phi d \theta,
\end{array}
$$

where $G(\phi)=\left(\int_{r=0}^{\infty} g(r \phi)^{p^{\prime}} r^{n} \frac{d r}{r}\right)^{1 / p^{\prime}}$. The bracketed expression in (2.2) is the $L^{p}$ norm of the group $\left(\mathbb{R}^{+}, \frac{d t}{t}\right)$ convolution of the function $t \rightarrow f(t \theta) t^{\frac{n}{p}}$ with the kernel $\chi_{[0, \theta \cdot \phi]}(t) t t^{\frac{n}{p^{\prime}}}$ at $2 r$. We therefore estimate $(2.2)$ by

$$
\frac{2{\frac{n}{p^{t}}}^{n}}{v_{n}} \iint_{\left(S^{n-1}\right)^{2}} G(\phi) F(\theta)\left(\int_{0}^{\theta \cdot \phi} t^{\frac{n}{p^{\prime}}} \frac{d t}{t}\right) d \phi d \theta
$$

where $F(\theta)=\left(\int_{0}^{\infty} f(r \theta)^{p} r^{n} \frac{d r}{r}\right)^{1 / p}$. Let

$$
K(\phi \cdot \theta)=\int_{0}^{\theta \cdot \phi} t^{n / p^{\prime}} \frac{d t}{t}=\frac{p^{\prime}}{n}\left[(\phi \cdot \theta)^{+}\right]^{n / p^{\prime}},
$$


where $N^{+}$denotes the positive part of the number $N$. Next, we need the following:

Lemma. For any $F, G \geq 0$ measurable on $S^{n-1}$ and $K \geq 0$ measurable on $[-1,1]$,

$$
\begin{aligned}
& \iint_{\left(S^{n-1}\right)^{2}} F(\theta) G(\phi) K(\theta \cdot \phi) d \phi d \theta \\
& \quad \leq\|F\|_{L^{p}\left(S^{n-1}\right)}\|G\|_{L^{p^{\prime}\left(S^{n-1}\right)}} \int_{S^{n-1}} K(\theta \cdot \phi) d \phi .
\end{aligned}
$$

Proof. We may assume that all three quantities on the right-hand side of (2.4) are finite. Since $K$ depends only on the inner product $\theta \cdot \phi$, the integral $\int_{S^{n-1}} K(\theta \cdot \phi) d \phi$ is independent of $\theta$. Hölder's inequality applied to the functions $F$ and 1 with respect to the measure $K(\theta \cdot \phi) d \theta$ gives

$$
\begin{array}{rl}
\int_{S^{n-1}} & F(\theta) K(\theta \cdot \phi) d \theta \\
& \leq\left(\int_{S^{n-1}} F(\theta)^{p} K(\theta \cdot \phi) d \theta\right)^{1 / p}\left(\int_{S^{n-1}} K(\theta \cdot \phi) d \theta\right)^{1 / p^{\prime}} .
\end{array}
$$

We will now use (2.5) to prove (2.4). The left-hand side of (2.4) is trivially estimated by $\left(\int_{S^{n-1}}\left(\int_{S^{n-1}} F(\theta) K(\theta \cdot \phi) d \theta\right)^{p} d \phi\right)^{1 / p}\|G\|_{L^{p^{\prime}}\left(S^{n-1}\right)}$. Applying (2.5) and Fubini's theorem we bound this last expression by $\|F\|_{L^{p}\left(S^{n-1}\right)}\|G\|_{L^{p^{\prime}}\left(S^{n-1}\right)} \times$ $\int_{S^{n-1}} K(\theta \cdot \phi) d \phi$. The lemma is now proved. Observe that equality is attained in (2.4) if and only if both $F$ and $G$ are constants.

We now continue with the proof of Theorem 2. Applying the lemma and using the fact that $F$ and $G$ have norm one, we estimate $(2.3)$ by $\frac{p^{\prime}}{n} \frac{2 p^{n}}{v_{n}} \times$ $\int_{S^{n-1}}\left((\theta \cdot \phi)^{+}\right)^{\frac{n}{p^{\prime}}} d \theta$. To compute this integral, we slice the sphere in the direction transverse to $\phi$. For convenience we may take $\phi=e_{1}=(1,0, \ldots, 0)$. The area of the slice cut by the hyperplane $\phi_{1}=s$ is $\omega_{n-2}\left(1-s^{2}\right)^{\frac{n-2}{2}}$ and the weight of this slice is $\left(1-s^{2}\right)^{-\frac{1}{2}}$. We get

$$
\begin{aligned}
\int_{S^{n-1}}\left((\theta \cdot \phi)^{+}\right)^{\frac{n}{p^{\prime}}} d \theta & =\omega_{n-2} \int_{s=0}^{1} s^{\frac{n}{p^{\prime}}}\left(1-s^{2}\right)^{\frac{n-3}{2}} d s \\
& =\omega_{n-2} \frac{1}{2} B\left(\frac{1}{2}\left(\frac{n}{p^{\prime}}-1\right), \frac{n-3}{2}\right) .
\end{aligned}
$$

We now use that $n v_{n}=\omega_{n-1}$ to get the final estimate $c_{p, n}$ in (2.2) which completes the proof of (2.1). It remains to establish that the constant $c_{p, n}$ is the best possible. For any $y \in \mathbb{R}^{n}$, let $A(y)$ be the spherical cap $\{\theta \in$ $\left.S^{n-1}:|\theta-y| \leq|y|\right\}$. This cap is nonempty if and only if $|y| \geq 1 / 2$. For such $y$, the Lebesgue measure $|A(y)|$ is $\omega_{n-2} \int_{1 / 2|y|}^{1}\left(1-s^{2}\right)^{\frac{n-3}{2}} d s$. Let $G(t)=$ $\chi_{[0,1]}(t) t^{n / p^{\prime}} \int_{t}^{1}\left(1-s^{2}\right)^{\frac{n-3}{2}} d s$. An easy computation shows that $\|G\|_{L^{1}\left(\mathbf{R}^{+}, \frac{d t}{t}\right)}=$ $\left(\frac{p^{\prime}}{n}\right) \int_{0}^{1}\left(1-s^{2}\right)^{\frac{n-3}{2}} s^{\frac{n}{p^{+}}} d s$. Let $h=h_{\epsilon, N}$ be an element of the family 
$|x|^{-n / p} \chi_{\epsilon \leq|x| \leq N}$ normalized to have $L^{p}$ norm one. We have

$$
\begin{aligned}
\|S h\|_{L^{p}\left(\mathbb{R}^{n}\right)}^{p} & =\int_{r=0}^{\infty} \int_{\phi \in S^{n-1}}\left(\frac{1}{v_{n} r^{n}} \int_{D(r \phi, r)} h(y) d y\right)^{p} r^{n-1} d \phi d r \\
& =\int_{r=0}^{\infty} \int_{\phi \in S^{n-1}}\left(\frac{1}{v_{n} r^{n}} \int_{t=0}^{2 r} \int_{t \theta \in S^{n-1}(r \phi, r)} h(t \theta) t^{n-1} d \theta d t\right)^{p} r^{n-1} d \phi d r \\
& =\int_{r=0}^{\infty} \int_{\phi \in S^{n-1}}\left(\frac{1}{v_{n} r^{n}} \int_{t=0}^{2 r}|A((r / t) \phi)| h(t) t^{n} \frac{d t}{t}\right)^{p} r^{n} d \phi \frac{d r}{r} \\
& =\omega_{n-2}^{p} \frac{2^{n p-n}}{v_{n}^{p}} \omega_{n-1} \int_{r=0}^{\infty}\left(\int_{t=0}^{1} h(2 r t)(2 r t)^{\frac{n}{p}} G(t) \frac{d t}{t}\right)^{p} r^{n} \frac{d r}{r} .
\end{aligned}
$$

The convolution inequality $\|g * L\|_{L^{p}} \leq\|g\|_{L^{p}}\|L\|_{L^{1}}$ in the group $\left(\mathbb{R}^{+}, \frac{d t}{t}\right)$ written as

$$
\int_{r=0}^{\infty}\left(\int_{t=0}^{1} h(2 r t)(2 r t)^{\frac{n}{p}} G(t) \frac{d t}{t}\right)^{p} \frac{d r}{r} \leq\left(\int_{r=0}^{\infty} h(r)^{p} r^{n} \frac{d r}{r}\right)\|G\|_{L^{1}\left(\mathbf{R}^{+}, \frac{d t}{t}\right)}^{p}
$$

becomes an equality as $\epsilon \rightarrow 0$ and $N \rightarrow \infty$. Inserting (2.8) in (2.7) we obtain

$$
\begin{aligned}
\|S h\|_{L^{p\left(\mathbb{R}^{n}\right)}}^{p} \leq & \omega_{n-2}^{p} \frac{2^{n p-n}}{v_{n}^{p}}\left(\frac{p^{\prime}}{n}\right)^{p}\left(\int_{s=0}^{1}\left(1-s^{2}\right)^{\frac{n-3}{2}} s^{\frac{n}{p^{\prime}}} d s\right)^{p} \\
& \times \omega_{n-1} \int_{r=0}^{\infty} h(r)^{p} r^{n-1} d r=c_{p, n}^{p}
\end{aligned}
$$

since $\|h\|_{L^{p}}=1$, and equality is attained as $\epsilon \rightarrow 0$ and $N \rightarrow \infty$. Theorem 2 is now proved.

3. A LOWER BOUND FOR THE OPERATOR NORM OF THE HARDY-LitTLEWOOD MAXIMAL FUNCTION ON $L^{p}\left(\mathbb{R}^{n}\right)$

Let $M(f)(x)=\sup _{r>0}\left(v_{n} r^{n}\right)^{-1} \int_{|y-x| \leq r}|f(y)| d y$ be the usual HardyLittlewood maximal function on $\mathbb{R}^{n}$. The family of functions $f_{\epsilon, N}(x)=$ $|x|^{-n / p} \chi_{\epsilon \leq|x| \leq N}$ is extremal for Theorems 1 and 2. Let $A_{p, n}$ be the operator norm of $M$ on $L^{p}\left(\mathbb{R}^{n}\right)$. By computing $\left\|M\left(f_{\epsilon, N}\right)\right\|_{L^{p}} /\left\|f_{\epsilon, N}\right\|_{L^{p}}$ and letting $\epsilon \rightarrow 0$ and $N \rightarrow \infty$ we obtain a lower bound for $A_{p, n}$.

Proposition. For $1<p<\infty$, let $A_{p, n}$ be the best constant $C$ that satisfies the inequality $\|M f\|_{L^{p}\left(\mathbb{R}^{n}\right)} \leq C\|f\|_{L^{p}\left(\mathbb{R}^{n}\right)}$ for all $f$ in $L^{p}$. Then

$$
A_{p, n} \geq p^{\prime} \frac{\omega_{n-2}}{\omega_{n-1}} \sup _{\delta>1} \frac{1}{\delta^{n}} \int_{-1}^{1}\left(\sqrt{1-s^{2}}\right)^{n-3}\left(s+\sqrt{s^{2}+\delta^{2}-1}\right)^{\frac{n}{p^{\prime}}} d s
$$

and the supremum above is attained for some $\delta=\delta_{n, p}$ always less than 2 .

Proof. The following is only a sketch. Since $|x|^{-n / p}$ is in $L_{\text {loc }}^{1}\left(\mathbb{R}^{n}\right)$, we can calculate $M\left(|x|^{-n / p}\right)$ instead. Observe that $M\left(|x|^{-n / p}\right)=c|x|^{-n / p}$ where $c=$ $M\left(|x|^{-n / p}\right)\left(e_{1}\right)$ and $e_{1}=(1,0, \ldots, 0)$. Also note that the supremum of the averages of $|x|^{-n / p}$ over balls of radius $r$ centered at $e_{1}$ is attained for some $r=1+\gamma$ where $\gamma>0$. We therefore find that

$$
c=\sup _{\gamma>0} \frac{1}{v_{n}(1+\gamma)^{n}} \int_{r=0}^{2+\gamma} r^{n-\frac{n}{p}} A_{r} \frac{d r}{r},
$$


where $A_{r}=\left|\left\{\theta \in S^{n-1}:\left|r \theta-e_{1}\right|<1+\gamma\right\}\right|$. Calculation gives that $A_{r}=\omega_{n-1}$ for $r \leq \gamma$ and $A_{r}=\omega_{n-2} \int_{\left(r^{2}-\gamma^{2}-2 \gamma\right) / 2 r}^{1}\left(1-s^{2}\right)^{\frac{n-3}{2}} d s$ for $2+\gamma>r>\gamma$. We plug these values into (3.2), and we interchange the. integration in $r$ and $s$ :

$$
\int_{r=\gamma}^{2+\gamma} \int_{s=\frac{r^{2}-\gamma^{2}-2 \gamma}{2 r}}^{1} r^{\frac{n}{p^{\prime}}}\left(1-s^{2}\right)^{\frac{n-3}{2}} d s \frac{d r}{r}=\int_{-1}^{1} \int_{r=\gamma}^{s+\sqrt{s^{2}+\gamma^{2}+2 \gamma}} r^{\frac{n}{p^{\prime}}}\left(1-s^{2}\right)^{\frac{n-3}{2}} \frac{d r}{r} d s .
$$

We now let $\delta=\gamma+1$ and obtain (3.1). Note that the constant on the right-hand side of (3.1) reduces to the constant $c_{p, n}$ of Theorem 2 when $\delta=1$.

\section{FinAL REMARKS}

We end with a couple of remarks. Let $c_{n, p}$ be the constant of Theorem 2 . We observe that $c_{n, p} \leq \frac{p}{p-1}$. This can be shown directly or via the following inequality which can be found in [HLP]:

$$
\int_{\mathbb{R}^{n}} f(x) g(x) d x \leq \int_{\mathbb{R}^{n}} \tilde{f}(x) \tilde{g}(x) d x,
$$

where $f$ and $g$ are integrable and $\tilde{f}$ denotes the symmetric decreasing rearrangement of any function $f$. Let $T$ and $S$ be the operators of Theorems 1 and 2. The nonsymmetric decreasing rearrangement of the kernel of $S$ is the kernel of $T$. Taking $g$ to be the kernel of $S$ and $f$ in $L^{p} \cap L^{1}$ in (4.1), we obtain the pointwise inequality $S f \leq T \tilde{f}$. It follows that $c_{n, p} \leq \frac{p}{p-1}$.

For any $\delta>0$, we define variants $T_{\delta}$ of $T$ and $S_{\delta}$ of $S$ as follows:

$$
\left(T_{\delta} f\right)(x)=\frac{1}{|D(0, \delta|x|)|} \int_{D(0, \delta|x|)} f(y) d y
$$

and

$$
\left(S_{\delta} f\right)(x)=\frac{1}{|D(x, \delta|x|)|} \int_{D(x, \delta|x|)} f(y) d y .
$$

Since $\left(T_{\delta} f\right)(x)=(T f)(\delta x)$, it is immediate that the operator norm of $T_{\delta}$ on $L^{p}\left(\mathbb{R}^{n}\right)$ is $\frac{p}{p-1} \delta^{-n / p}$.

To compute the operator norm of $S_{\delta}$ on $L^{p}\left(\mathbb{R}^{n}\right)$, we repeat the proof of Theorem 2 verbatim. We obtain the following result:

Theorem. (A) For $\delta>1$, the operator norm of $S_{\delta}$ on $L^{p}\left(\mathbb{R}^{n}\right)$ is

$$
p^{\prime} \frac{\omega_{n-2}}{\omega_{n-1}} \frac{1}{\delta^{n}} \int_{-1}^{1}\left(1-s^{2}\right)^{\frac{n-3}{2}}\left(s+\sqrt{s^{2}+\delta^{2}-1}\right)^{\frac{n}{p^{\prime}}} d s .
$$

(B) For $\delta<1$, the operator norm of $S_{\delta}$ on $L^{p}\left(\mathbb{R}^{n}\right)$ is

$p^{\prime} \frac{\omega_{n-2}}{\omega_{n-1}} \frac{1}{\delta^{n}} \int_{s=\sqrt{1-\delta^{2}}}^{1}\left(1-s^{2}\right)^{\frac{n-3}{2}}\left[\left(s+\sqrt{s^{2}+\delta^{2}-1}\right)^{\frac{n}{p^{\prime}}}-\left(s-\sqrt{s^{2}+\delta^{2}-1}\right)^{\frac{n}{p^{\prime}}}\right] d s$.

(3.1) is of course subsumed in conclusion (A) above.

\section{ACKNOWLEDGMENT}

The second author would like to thank Professor Al Baernstein for stimulating his interest in these problems and also for many useful conversations. 


\section{REFERENCES}

[BT] A. Baernstein II and B. A. Taylor, Spherical rearrangements, subharmonic functions and *-functions in n-space, Duke Math. J. 43 (1976), 245-268.

[HLP] G. Hardy, J. Littlewood, and G. Pólya, Inequalities, The University Press, Cambridge, 1959.

[F] W. G. Faris, Weak Lebesgue spaces and quantum mechanical binding, Duke Math. J. 43 (1976), 365-373.

[PS] G. Pólya and G. Szegö, Isoperimatric inequalities in mathematical physics, Princeton Univ. Press, Princeton, NJ, 1951.

[S] E. M. Stein, Singular integrals and differentiability properties of functions, Princeton Univ. Press, Princeton, NJ, 1970.

[SO] S. L. Sobolev, On a theorem of functional analysis, Mat. Sb. (N.S.) 4 (1938), 471-497; English transl., Amer. Math. Soc. Transl. Ser. 2, vol. 34, Amer. Math. Soc., Providence, RI, 1963, pp. 39-68.

[SS] E. M. Stein and J. O. Strömberg, Behavior of maximal functions in $\mathbb{R}^{n}$ for large $n$, Ark. Mat. 21 (1983), 259-269.

[SW] E. M. Stein and G. Weiss, Introduction to Fourier analysis on Euclidean spaces, Princeton Univ. Press, Princeton, NJ, 1971.

Department of Mathematics, University of California-Los Angeles, Los Angeles, CALIFORNIA 90024-1555

E-mail address: christ@math.ucla.edu

Department of Mathematics, Washington University, St. Louis, Missouri 63130-4899

Current address: Department of Mathematics, University of Missouri-Columbia, Columbia, Missouri 65211

E-mail address: loukas@msindy3.cs.missouri.edu 